\title{
AUTOMORPHISM GROUP OF THE TERNARY SELF-DUAL CODE OF LENGTH 8
}

\author{
Hyun Jin Kim And June BoK LeE
}

\begin{abstract}
We study the abstract structure of the automorphism group of the ternary self-dual code of length 8 and give its convenient presentation by generators.
\end{abstract}

\section{Introduction}

An $[n, k]$ linear code $\mathcal{C}$ over the finite field $\mathbb{F}_{3}$ is a $k$-dimensional subspace of $\mathbb{F}_{3}^{n}$. The Hamming weight of a vector in $\mathbb{F}_{3}^{n}$ is the number of its nonzero coordinates. The minimum weight $d$ of $\mathcal{C}$ is the minimum weight of its nonzero codewords and in this case $\mathcal{C}$ is called an $[n, k, d]$ code. For every $u=\left(u_{1}, u_{2}, \ldots, u_{n}\right)$ and $v=\left(v_{1}, v_{2}, \ldots, v_{n}\right)$ from $\mathbb{F}_{3}^{n}, u \cdot v=u_{1} \cdot v_{1}+u_{2} \cdot v_{2}+\cdots+u_{n} \cdot v_{n}$ defines the Euclidean inner product in $\mathbb{F}_{3}^{n}$. The dual code of $\mathcal{C}$ is defined by $\mathcal{C}^{\perp}=\left\{u \in \mathbb{F}_{2}^{n}: u \cdot v=0\right.$ for all $\left.v \in \mathcal{C}\right\}$, and $\mathcal{C}^{\perp}$ is a linear $[n, n-k]$ code. If $\mathcal{C} \subseteq \mathcal{C}^{\perp}$, then $\mathcal{C}$ is called self-orthogonal, and if $\mathcal{C}=\mathcal{C}^{\perp}$, then we call it selfdual. Self-dual codes over $\mathbb{F}_{3}$ exist only for lengths a multiple of 4 and have codewords of Hamming weight a multiple of 3 . Self-dual codes with the largest minimum weight of given length are called optimal. Extremal self-dual codes have minimum distance $3[n / 12]+3([3])$. The largest possible minimum weight of the self-dual codes of lengths $n=4$ and 8 is 3 .

When we consider code classification, a notion of equivalence is necessary. An $n \times n$ matrix with coefficients in $\mathbb{F}_{3}$ is called monomial if there is exactly one nonzero entry in each row and column. Such a matrix is invertible since all nonzero elements of $\mathbb{F}_{3}$ are invertible. If all nonzero entries of the monomial are 1 , then it is said to be a permutation matrix. Any monomial matrix can be uniquely written as the product of a permutation matrix and diagonal matrix. A monomial matrix $M$ acts on the elements $x \in \mathbb{F}_{3}^{n}$ as $M \cdot x=x M$. Two codes $\mathcal{C}_{1}$ and $\mathcal{C}_{2}$ are permutation equivalent if there exists a permutation matrix $P$

Received March 14, 2011.

2010 Mathematics Subject Classification. Primary 20H30, $94 \mathrm{~B} 05$.

Key words and phrases. automorphism group, ternary self-dual code.

The first author was supported by Priority Research Centers Program through the National Research Foundation of Korea(NRF) funded by the Ministry of Education, Science and Technology(2012-0006691). 
such that $\mathcal{C}_{1}=\mathcal{C}_{2} P$. More generally, if there is a monomial matrix $M$ such that $\mathcal{C}_{1}=\mathcal{C}_{2} M$, the codes $\mathcal{C}_{1}$ and $\mathcal{C}_{2}$ are equivalent. The automorphism group of a ternary code $\mathcal{C}$ is the set of all monomial matrices $M$ such that $\mathcal{C}=\mathcal{C} M$. Characteristics for ternary self-dual codes were given in $[2,5]$.

Classification of self-dual codes over a ring requires not only the size of automorphism groups of codes over a field as well as properties of their subgroups and their relationshilp. Recently, the automorphism group of the ternary tetracode is presented in detail by the author in [4]. In this present paper, we extend the automorphism group in the case of the extremal self-dual code of length 8 over $\mathbb{F}_{3}$.

\section{Preliminaries}

The classification of self-dual codes relies on the knowledge of the so-called counting formula for self-dual codes, and the size of automorphism groups. The following counting formula for ternary codes of length $n$ is well-known in [5].

Lemma 2.1 ([5]). There exists a ternary self-dual code of length $n$ if and only if $n$ is divisible by 4. In this case, the number of self-dual code of length $n$ is given by

$$
2 \prod_{i=1}^{\frac{n}{2}-1}\left(3^{i}+1\right) .
$$

Suppose that $\mathcal{C}_{1}, \mathcal{C}_{2}, \ldots, \mathcal{C}_{m}$ are all inequivalent ternary self-dual codes of length $n$. Then

$$
2 \prod_{i=1}^{\frac{n}{2}-1}\left(3^{i}+1\right)=\sum_{j=1}^{m} \frac{\left|M_{n}\left(\mathbb{Z}_{3}\right)\right|}{\left|\operatorname{Aut}\left(\mathcal{C}_{j}\right)\right|} .
$$

The tetracode is a ternary code $\mathcal{T}$ with generator matrix

$$
\left(\begin{array}{llll}
1 & 0 & 1 & 1 \\
0 & 1 & 1 & 2
\end{array}\right)
$$

Any self-dual code of length 4 is equivalent to $\mathcal{T}$. From $(1), \operatorname{Aut}(\mathcal{T})$ has order 48.

Lemma 2.2 ([4, Theorem 2.1]). The automorphism group of $\mathcal{T}$ can be generated by two elements $\mathbf{b}$ and $\mathbf{c}$, where

$$
\mathbf{b}=\left(\begin{array}{cccc}
0 & 0 & 0 & -1 \\
1 & 0 & 0 & 0 \\
0 & 1 & 0 & 0 \\
0 & 0 & 1 & 0
\end{array}\right), \quad \mathbf{c}=\left(\begin{array}{cccc}
0 & 0 & 0 & 1 \\
0 & 1 & 0 & 0 \\
1 & 0 & 0 & 0 \\
0 & 0 & 1 & 0
\end{array}\right)
$$

This lemma is used to identify all elements of $\operatorname{Aut}(\mathcal{T})$. We can see them on the Table 1 in [4]. 
Let

$$
\begin{aligned}
\mathbf{i}=\left(\begin{array}{cccc}
0 & 0 & 0 & 1 \\
0 & 0 & -1 & 0 \\
0 & 1 & 0 & 0 \\
-1 & 0 & 0 & 0
\end{array}\right), \mathbf{j}=\left(\begin{array}{cccc}
0 & 0 & -1 & 0 \\
0 & 0 & 0 & -1 \\
1 & 0 & 0 & 0 \\
0 & 1 & 0 & 0
\end{array}\right) \\
\mathbf{d}=\left(\begin{array}{cccc}
-1 & 0 & 0 & 0 \\
0 & 0 & 0 & -1 \\
0 & 0 & 1 & 0 \\
0 & -1 & 0 & 0
\end{array}\right)
\end{aligned}
$$

These generate the unique normal subgroup of $\operatorname{Aut}(\mathcal{T})$, and $\mathbf{i}, \mathbf{j}, \mathbf{c}$, and $\mathbf{d}$ give a presentation of $\operatorname{Aut}(\mathcal{T})[4]$.

Lemma 2.3 ([4, Theorem 3.6]). The automorphism group of $\mathcal{T}$ can be expressed by:

$$
\operatorname{Aut}(\mathcal{T})=\left\{\mathbf{i}^{i} \mathbf{j}^{j} \mathbf{c}^{c} \mathbf{d}^{d} \mid 0 \leq i \leq 3,0 \leq j \leq 1,0 \leq c \leq 2,0 \leq d \leq 1\right\}
$$

Let $G$ be a group and $A$ be a subgroup of $G$. The normalizer of $A$ in $G$ is denoted by $N_{G}(A)$. In [4], we see three Sylow 2-subgroups of order 16

$$
\begin{gathered}
P_{1}=N_{\text {Aut }(\mathcal{T})}\left(\left(\begin{array}{cccc}
0 & 1 & 0 & 0 \\
0 & 0 & 1 & 0 \\
0 & 0 & 0 & 1 \\
-1 & 0 & 0 & 0
\end{array}\right)\right), \\
P_{2}=N_{\text {Aut }(\mathcal{T})}\left(\left(\begin{array}{cccc}
0 & 0 & 1 & 0 \\
1 & 0 & 0 & 0 \\
0 & 0 & 0 & -1 \\
0 & 1 & 0 & 0
\end{array}\right)\right) \\
P_{3}=N_{\text {Aut }(\mathcal{T})}\left(\left(\begin{array}{cccc}
0 & 0 & 0 & -1 \\
0 & 0 & -1 & 0 \\
1 & 0 & 0 & 0 \\
0 & -1 & 0 & 0
\end{array}\right)\right)
\end{gathered}
$$

and four Sylow 3-subgroups of order 3

$$
\begin{gathered}
Q_{1}=N_{\text {Aut }(\mathcal{T})}\left(\left(\begin{array}{cccc}
1 & 0 & 0 & 0 \\
0 & 0 & 1 & 0 \\
0 & 0 & 0 & -1 \\
0 & -1 & 0 & 0
\end{array}\right)\right), Q_{2}=N_{\operatorname{Aut}(\mathcal{T})}\left(\left(\begin{array}{cccc}
0 & 1 & 0 & 0 \\
0 & 0 & -1 & 0 \\
-1 & 0 & 0 & 0 \\
0 & 0 & 0 & 1
\end{array}\right)\right), \\
Q_{3}=N_{\operatorname{Aut}(\mathcal{T})}\left(\left(\begin{array}{llll}
0 & 0 & 1 & 0 \\
0 & 1 & 0 & 0 \\
0 & 0 & 0 & 1 \\
1 & 0 & 0 & 0
\end{array}\right)\right), Q_{4}=N_{\operatorname{Aut}(\mathcal{T})}\left(\left(\begin{array}{cccc}
0 & 0 & 0 & -1 \\
-1 & 0 & 0 & 0 \\
0 & 0 & 1 & 0 \\
0 & 1 & 0 & 0
\end{array}\right)\right) .
\end{gathered}
$$




\subsection{Automorphism on ternary $[8,4,3]$ code}

Let $\mathcal{C}$ be a ternary $[8,4,3]$ code with the following generator matrix

$$
\left(\begin{array}{llllllll}
1 & 0 & 1 & 1 & 0 & 0 & 0 & 0 \\
0 & 1 & 1 & 2 & 0 & 0 & 0 & 0 \\
0 & 0 & 0 & 0 & 1 & 0 & 1 & 1 \\
0 & 0 & 0 & 0 & 0 & 1 & 1 & 2
\end{array}\right)
$$

and let us define $\mathbf{G}=\operatorname{Aut}(\mathcal{C})$. Then this is a unique self-dual code of length 8 up to equivalence. From (1), we know that

$$
2 \times(3+1) \times(9+1) \times(27+1)=\frac{2^{8} \cdot 8 !}{|\mathbf{G}|} .
$$

In this case, the order of $\mathbf{G}$ is 4608 . We define the maps

$$
\phi_{1}: \operatorname{Aut}(\mathcal{T}) \rightarrow \mathbf{G} \text { and } \phi_{2}: \operatorname{Aut}(\mathcal{T}) \rightarrow \mathbf{G}
$$

by $\phi_{1}(\sigma)=\left(\begin{array}{cc}\sigma & 0 \\ \mathbf{0} & I_{4}\end{array}\right)$ and $\phi_{2}(\sigma)=\left(\begin{array}{cc}I_{4} & \mathbf{0} \\ \mathbf{0} & \sigma\end{array}\right)$ for $\sigma \in \operatorname{Aut}(\mathcal{T})$, where $I_{4}$ is the $4 \times 4$ identity matrix and $\mathbf{0}$ is the $4 \times 4$ zero matrix. Obviously, $\phi_{1}(\operatorname{Aut}(\mathcal{T}))$ and $\phi_{2}(\operatorname{Aut}(\mathcal{T}))$ are subgroups of $\mathbf{G}$. For any subgroup $A$ of $\mathbf{G}$, the centralizer of $A$ in $\mathbf{G}$ is denoted by $C_{\mathbf{G}}(A)$. Let $\mathbf{G}_{1}=\phi_{1}(\operatorname{Aut}(\mathcal{T}))$ and $\mathbf{G}_{2}=\phi_{2}(\operatorname{Aut}(\mathcal{T}))$. Then $\mathbf{G}_{1} \leq C_{\mathbf{G}}\left(\mathbf{G}_{2}\right)$ and $\mathbf{G}_{2} \leq C_{\mathbf{G}}\left(\mathbf{G}_{1}\right)$, that is, $\bar{\sigma}_{1} \bar{\sigma}_{2}=\bar{\sigma}_{2} \bar{\sigma}_{1}$ for any $\bar{\sigma}_{1} \in \mathbf{G}_{1}$, $\bar{\sigma}_{2} \in \mathbf{G}_{2}$.

Lemma 2.4. Let $Z$ be a group. If $X$ and $Y$ are subgroups of $Z$, then $X Y$ is a subgroup of $Z$ if and only if $X Y=Y X$.

Proof. Suppose $X Y$ is a subgroup of $Z$. Then for any $x \in X, y \in Y$,

$$
y x X Y=y X Y=x x^{-1} y X Y=x X Y=X Y \Rightarrow y x \in X Y .
$$

Since the order of $X Y$ is equal to the number of elements of $Y X$, we have $X Y=Y X$.

For the other direction, now suppose $X Y=Y X$. For any $z, z^{\prime} \in Y X$, $z=x_{1} y_{1}$ and $z^{\prime}=x_{2} y_{2}$ for some $x_{1}, x_{2} \in X$ and $y_{1}, y_{2} \in Y$.

$$
z^{-1} z^{\prime}=\left(x_{1} y_{1}\right)^{-1} x_{2} y_{2}=y_{1}^{-1} x_{1}^{-1} x_{2} y_{2} \text {. }
$$

Since $X$ is a group, $x_{1}^{-1} x_{2} \in X$. Let $x_{3}=x_{1}^{-1} x_{2}$. Then we have

$$
y_{1}^{-1} x_{1}^{-1} x_{2} y_{2}=y_{1}^{-1} x_{3} y_{2} \text {. }
$$

From the hypothesis, $y_{1}^{-1} x_{3}=x_{4} y_{3}$ for some $x_{4} \in X, y_{3} \in Y$, and so

$$
y_{1}^{-1} x_{3} y_{2}=x_{4} y_{3} y_{2} \text {. }
$$

Since $Y$ is a group, $y_{3} y_{2} \in Y$. Let $y_{4}=y_{3} y_{2}$. Then we have

$$
x_{4} y_{3} y_{2}=x_{4} y_{4} \in X Y \text {. }
$$

Therefore $X Y$ is a subgroup of $Z$.

The following theorem shows the abstract structure of $\mathbf{G}$. 
Theorem 2.5. The automorphism group of $\mathcal{C}$ can be expressed by the product of $\mathbf{G}_{1}, \mathbf{G}_{2}$, and $\mathbf{G}_{3}$, where $\mathbf{G}_{3}=\left\{I_{8}, \gamma\right\}$ with $\gamma=\left(\begin{array}{cc}\mathbf{0} & I_{4} \\ I_{4} & \mathbf{0}\end{array}\right)$.

Proof. It is known that $\mathbf{G}_{1} \leq C_{\mathbf{G}}\left(\mathbf{G}_{2}\right)$ implies $\mathbf{G}_{1} \mathbf{G}_{2}=\mathbf{G}_{2} \mathbf{G}_{1}$. From Lemma $2.4, \mathbf{G}_{1} \mathbf{G}_{2}$ is a subgroup of $\mathbf{G}$. Since the index of $\mathbf{G}_{1} \mathbf{G}_{2}$ in $\mathbf{G}$ is $2, \mathbf{G}_{1} \mathbf{G}_{2}$ is a normal subgroup of $\mathbf{G}$. Let $\gamma=\left(\begin{array}{cc}\mathbf{0} & I_{4} \\ I_{4} & \mathbf{0}\end{array}\right)$. It is easy to check that $\gamma \in \mathbf{G}$. Let $\mathbf{G}_{3}=\left\{I_{8}, \gamma\right\}$. Then $\mathbf{G}_{3} \leq N_{\mathbf{G}}\left(\mathbf{G}_{1} \mathbf{G}_{2}\right)$, and by the result of Lemma 2.4, we know that $\mathbf{G}_{1} \mathbf{G}_{2} \mathbf{G}_{3}$ is a subgroup of $\mathbf{G}$. Each order of $\mathbf{G}_{1}$ and $\mathbf{G}_{2}$ is 48 , and $\mathbf{G}_{1} \cap \mathbf{G}_{2}=\left\{I_{8}\right\}$. The order $\mathbf{G}_{3}$ is 2 , and $\mathbf{G}_{1} \mathbf{G}_{2} \cap \mathbf{G}_{3}=\left\{I_{8}\right\}$. Since $\left|\mathbf{G}_{1} \mathbf{G}_{2} \mathbf{G}_{3}\right|=4608$, we get the desired result: $\mathbf{G}=\mathbf{G}_{1} \mathbf{G}_{2} \mathbf{G}_{3}$.

The group $\mathbf{G}_{1} \mathbf{G}_{2}$ is isomorphic to $\operatorname{Aut}(\mathcal{T}) \times \operatorname{Aut}(\mathcal{T})$. Lemma 2.2 gives the following corollary. This corollary identifies all elements of $\mathbf{G}$.

Corollary 2.6. The automorphism group of $\mathcal{C}$ is generated by $\phi_{1}(\mathbf{b}), \phi_{1}(\mathbf{c})$, and $\gamma$.

Proof. From Lemma 2.2 and Theorem 2.5, $\phi_{1}(\mathbf{b}), \phi_{1}(\mathbf{c}), \phi_{2}(\mathbf{b}), \phi_{2}(\mathbf{c})$, and $\gamma$ generate G. Clearly,

$$
\phi_{2}(\mathbf{b})=\phi_{1}(\mathbf{b}) \gamma, \quad \phi_{2}(\mathbf{c})=\phi_{1}(\mathbf{c}) \gamma .
$$

Therefore, $\phi_{1}(\mathbf{b}), \phi_{1}(\mathbf{c})$, and $\gamma$ generate $\mathbf{G}$.

From Lemma 2.3 we can obtain the following corollary.

Corollary 2.7. The automorphism group of $\mathcal{C}$ can be presented by:

$$
\mathbf{G}=\left\{\phi_{1}\left(\mathbf{i}^{i} \mathbf{j}^{j} \mathbf{c}^{c} \mathbf{d}^{d}\right) \phi_{2}\left(\mathbf{i}^{i^{\prime}} \mathbf{j}^{j^{\prime}} \mathbf{c}^{c^{\prime}} \mathbf{d}^{d^{\prime}}\right) \gamma^{k} \mid \begin{array}{l}
0 \leq i, i^{\prime} \leq 3,0 \leq j, j^{\prime} \leq 1,0 \leq c, c^{\prime} \leq 2, \\
\\
\left.0 \leq d, d^{\prime} \leq 1,0 \leq k \leq 1,\right\}
\end{array}\right.
$$

Let $g, g^{\prime} \in \mathbf{G}$ with $g=\left(\begin{array}{cc}\sigma_{1} & \mathbf{0} \\ \mathbf{0} & \sigma_{2}\end{array}\right), g^{\prime}=\left(\begin{array}{cc}\mathbf{0} & \sigma_{1} \\ \sigma_{2} & \mathbf{0}\end{array}\right)$ for $\sigma_{1}, \sigma_{2} \in \operatorname{Aut}(\mathcal{T})$. Then

$$
\gamma g \gamma^{-1}=\left(\begin{array}{cc}
\sigma_{2} & \mathbf{0} \\
\mathbf{0} & \sigma_{1}
\end{array}\right), \gamma g^{\prime} \gamma^{-1}=\left(\begin{array}{cc}
\mathbf{0} & \sigma_{2} \\
\sigma_{1} & \mathbf{0}
\end{array}\right) .
$$

Proposition 2.8. Let $A$ be a subgroup of $\mathbf{G}$ with $\gamma \in A$. If $g, g^{\prime} \in A$, where $g=$ $\left(\begin{array}{cc}\sigma_{1} & \mathbf{0} \\ \mathbf{0} & \sigma_{2}\end{array}\right)$ and $g^{\prime}=\left(\begin{array}{cc}\mathbf{0} & \sigma_{1} \\ \sigma_{2} & \mathbf{0}\end{array}\right)$, then $A$ contains $\left(\begin{array}{cc}\sigma_{2} & \mathbf{0} \\ \mathbf{0} & \sigma_{1}\end{array}\right)$ and $\left(\begin{array}{cc}\mathbf{0} & \sigma_{2} \\ \sigma_{1} & \mathbf{0}\end{array}\right)$. In particular, if $A=A_{1} A_{2} G_{3}$ for some $A_{1} \leq \mathbf{G}_{1}, A_{2} \leq \mathbf{G}_{2}$, then $\phi_{1}^{-1}\left(A_{1}\right)=\phi_{2}^{-1}\left(A_{2}\right)$.

We compute the conjugacy classes of $\mathbf{G}$. First we consider the conjugacy classes of elements of $\mathbf{G}_{1} \mathbf{G}_{2}$.

Proposition 2.9. If $g \in \mathbf{G}_{1} \mathbf{G}_{2}$, then the conjugates of $g$ belongs to $\mathbf{G}_{1} \mathbf{G}_{2}$.

Proof. For any $a \in \mathbf{G}_{1} \mathbf{G}_{2}$, aga $a^{-1} \in \mathbf{G}_{1} \mathbf{G}_{2}$ since $\mathbf{G}_{1} \mathbf{G}_{2}$ is a subgroup of $\mathbf{G}$, and in addition

$$
(a \gamma) g(a \gamma)^{-1}=a \gamma g \gamma^{-1} a^{-1}=a\left(\gamma g \gamma^{-1}\right) a^{-1} .
$$

Since $\left(\gamma g \gamma^{-1}\right) \in \mathbf{G}_{1} \mathbf{G}_{2}$ from Proposition 2.8, $(a \gamma) g(a \gamma)^{-1}$ belongs to $\mathbf{G}_{1} \mathbf{G}_{2}$. 
This shows that the conjugacy classes of elements of $\mathbf{G}_{1} \mathbf{G}_{2}$ are closed in $\mathbf{G}_{1} \mathbf{G}_{2}$. The following proposition gives the property which the conjugacy classes of elements of $\mathbf{G}_{1} \mathbf{G}_{2}$ have.

Proposition 2.10. For $\sigma_{1}, \sigma_{2} \in \operatorname{Aut}(\mathcal{T}), \phi_{1}\left(\sigma_{1}\right) \phi_{2}\left(\sigma_{2}\right)$ and $\phi_{1}\left(\sigma_{2}\right) \phi_{2}\left(\sigma_{1}\right)$ are affiliated to same conjugacy class.

Proof. From Proposition 2.8, we have

$$
\gamma \phi_{1}\left(\sigma_{1}\right) \phi_{2}\left(\sigma_{2}\right) \gamma^{-1}=\phi_{1}\left(\sigma_{2}\right) \phi_{2}\left(\sigma_{1}\right) \text {. }
$$

Therefore, $\phi_{1}\left(\sigma_{2}\right) \phi_{2}\left(\sigma_{1}\right)$ is a conjugate of $\phi_{1}\left(\sigma_{1}\right) \phi_{2}\left(\sigma_{2}\right)$.

The following theorem presents the conjugacy classes of elements of $\mathbf{G}_{1} \mathbf{G}_{2}$ clearly. We can see conjugacy classes of $\operatorname{Aut}(\mathcal{T})$ in [Table 2, [4]].

Theorem 2.11. For $\sigma_{i}, \sigma_{j} \in \operatorname{Aut}(\mathcal{T})$, the conjugacy class of $\phi_{1}\left(\sigma_{i}\right) \phi_{2}\left(\sigma_{j}\right)$ is the union of $\phi_{1}(C i) \phi_{2}(C j)$ and $\phi_{1}(C j) \phi_{2}(C i)$ where $C i$ and $C j$ are the conjugacy classes of $\sigma_{i}$ and $\sigma_{j}$ in $\operatorname{Aut}(\mathcal{T})$, respectively.

Proof. Let $a=\left(\begin{array}{ll}x & 0 \\ 0 & y\end{array}\right)$ with $x, y \in \operatorname{Aut}(\mathcal{T})$. Then since $a^{-1}=\left(\begin{array}{cc}x^{-1} & 0 \\ 0 & y^{-1}\end{array}\right)$,

$$
a\left(\begin{array}{cc}
\sigma_{i} & 0 \\
0 & \sigma_{j}
\end{array}\right) a^{-1}=\left(\begin{array}{cc}
x \sigma_{i} x^{-1} & 0 \\
0 & y \sigma_{j} y^{-1}
\end{array}\right) \in \phi_{1}(C i) \phi_{2}(C j),
$$

and

$$
\begin{aligned}
r l(a \gamma)\left(\begin{array}{cc}
\sigma_{i} & 0 \\
0 & \sigma_{j}
\end{array}\right)(a \gamma)^{-1} & =a\left(\gamma\left(\begin{array}{cc}
\sigma_{i} & 0 \\
0 & \sigma_{j}
\end{array}\right) \gamma^{-1}\right) a^{-1} \\
& =a\left(\begin{array}{cc}
\sigma_{j} & 0 \\
0 & \sigma_{i}
\end{array}\right) a^{-1} \in \phi_{1}(C j) \phi_{2}(C i) .
\end{aligned}
$$

Therefore, the union of $\phi_{1}(C i) \phi_{2}(C j)$ and $\phi_{1}(C j) \phi_{2}(C i)$ contains the conjugacy class of $\phi_{1}\left(\sigma_{i}\right) \phi_{2}\left(\sigma_{j}\right)$. On the other hand, since the conjugacy class of $\phi_{1}\left(\sigma_{i}\right) \phi_{2}\left(\sigma_{j}\right)$ contains $\phi_{1}(C i) \phi_{2}(C j)$, the conjugacy class of $\phi_{1}\left(\sigma_{i}\right) \phi_{2}\left(\sigma_{j}\right)$ includes $\phi_{1}(C j) \phi_{2}(C i)$ from Proposition 2.10. We get the desired result: the conjugacy class of $\phi_{1}\left(\sigma_{i}\right) \phi_{2}\left(\sigma_{j}\right)$ is equal to the union of $\phi_{1}(C i) \phi_{2}(C j)$ and $\phi_{1}(C j) \phi_{2}(C i)$.

We consider the conjugacy class of $a \gamma$ where $a$ is any element of $\mathbf{G}_{1} \mathbf{G}_{2}$.

Theorem 2.12. The conjugacy class of $\gamma$ is the set

$$
\left\{\left(\begin{array}{cc}
0 & \sigma \\
\sigma^{-1} & 0
\end{array}\right) \mid \sigma \in \operatorname{Aut}(\mathcal{T})\right\} .
$$

Proof. Let $a \gamma$ be any element of $\mathbf{G}$ with $a \in \mathbf{G}_{1} \mathbf{G}_{2}$. Then one has

$$
(a \gamma) \gamma(a \gamma)^{-1}=(a \gamma) \gamma\left(\gamma^{-1} a^{-1}\right)=a\left(\gamma \gamma \gamma^{-1}\right) a^{-1}=a \gamma a^{-1} .
$$


For any $x, y \in \operatorname{Aut}(\mathcal{T})$,

$$
\begin{aligned}
r l\left(\begin{array}{ll}
x & 0 \\
0 & y
\end{array}\right) \gamma\left(\begin{array}{cc}
x^{-1} & 0 \\
0 & y^{-1}
\end{array}\right) & =\left(\begin{array}{ll}
x & 0 \\
0 & y
\end{array}\right)\left(\begin{array}{cc}
0 & 1 \\
1 & 0
\end{array}\right)\left(\begin{array}{cc}
x^{-1} & 0 \\
0 & y^{-1}
\end{array}\right) \\
& =\left(\begin{array}{cc}
0 & x y^{-1} \\
y x^{-1} & 0
\end{array}\right) \\
& =\left(\begin{array}{cc}
0 & x y^{-1} \\
\left(x y^{-1}\right)^{-1} & 0
\end{array}\right) .
\end{aligned}
$$

Since all elements of $\operatorname{Aut}(\mathcal{T})$ can be presented as the form of $x y^{-1}$, this proof is complete.

From this, we know that the length of the conjugacy class of $\gamma$ is 48 .

Proposition 2.13. Let $\bar{\gamma}=y^{-1} \gamma y$ where $y$ is any element of $\mathbf{G}$. Then the conjugates of a $\gamma$ are equal to the conjugates of $y^{-1} a y \bar{\gamma}$.

Proof. For any $x \in \mathbf{G}$,

$$
\begin{aligned}
x a \gamma x^{-1} & =x a\left(y \bar{\gamma} y^{-1}\right) x^{-1} \\
& =x\left(y y^{-1}\right) a\left(y \bar{\gamma} y^{-1}\right) x^{-1} \\
& =(x y)\left(y^{-1} a y\right) \bar{\gamma}\left(y^{-1} x^{-1}\right) \\
& =(x y)\left(y^{-1} a y\right) \bar{\gamma}(x y)^{-1} .
\end{aligned}
$$

This proves that the conjugates of $a \gamma$ and $y^{-1} a y \bar{\gamma}$ are the same.

Proposition 2.14. The conjugacy class of a $\gamma$ is contained in the set $C_{a} C_{\gamma}$ where $C_{a}$ is the conjugacy class of $a$ and $C_{\gamma}$ is the conjugacy class of $\gamma$.

Proof. Let $C_{a \gamma}$ be the conjugacy class of $a \gamma$. Then any element $g$ of $C_{a \gamma}$ is representative as $b(a \gamma) b^{-1}$ for some $b \in \mathbf{G}$.

$$
\begin{aligned}
g & =b(a \gamma) b^{-1} \\
& =b\left\{a\left(b^{-1} b\right) \gamma\right\} b^{-1} \\
& =\left(b a b^{-1}\right)\left(b \gamma b^{-1}\right) .
\end{aligned}
$$

Consequently, $g \in C_{a} C_{\gamma}$ since $b a b^{-1} \in C_{a}$ and $b \gamma b^{-1} \in C_{\gamma}$.

These two propositions characterize conditions the conjugacy classes of elements which have $\gamma$ as the factor should satisfy.

If $h$ is an element in $C_{a} C_{\gamma}$, then there are some $b$ and $d$ in $\mathbf{G}$ such that $h=\left(b a b^{-1}\right)\left(d \gamma d^{-1}\right)$.

Proposition 2.15. If $h \in C_{a} C_{\gamma}$, then $h=d\left\{\left(d^{-1} b\right) a\left(d^{-1} b\right)^{-1}\right\} \gamma d^{-1}$. If $d^{-1} b$ belongs to centralizer of a, then the element $h$ of $C_{a} C_{\gamma}$ is contained in $C_{a \gamma}$. 
Proof. By the property of identity and the association law, one has

$$
\begin{aligned}
h & =\left(b a b^{-1}\right)\left(d \gamma d^{-1}\right) \\
& =\left(d d^{-1}\right)\left(b a b^{-1}\right)\left(d \gamma d^{-1}\right) \\
& =d\left(d^{-1} b a b^{-1} d\right) \gamma d^{-1} \\
& =d\left\{\left(d^{-1} b\right) a\left(b^{-1} d\right)\right\} \gamma d^{-1} \\
& =d\left\{\left(d^{-1} b\right) a\left(d^{-1} b\right)^{-1}\right\} \gamma d^{-1} .
\end{aligned}
$$

Let us consider the conjugacy classes of special elements of $\mathbf{G}$.

Theorem 2.16. The conjugacy class of $\phi_{1}(\sigma) \phi_{2}\left(I_{4}\right) \gamma$ is equal to $C_{\sigma} C_{\gamma}$, where $C_{\sigma}$ is the conjugacy class of $\phi_{1}(\sigma) \phi_{2}\left(I_{4}\right)$ and $C_{\gamma}$ is the conjugacy class of $\gamma$.

Proof. From Proposition 2.14 the conjugacy class of $\phi_{1}(\sigma) \phi_{2}\left(I_{4}\right) \gamma$ is contained in $C_{\sigma} C_{\gamma}$. Let $a$ be an element of $C_{\sigma} C_{\gamma}$. Then

$$
a=\left(\begin{array}{cc}
x \sigma x^{-1} & 0 \\
0 & I_{4}
\end{array}\right)\left(\begin{array}{cc}
0 & y \\
y^{-1} & 0
\end{array}\right) \quad \text { or } \quad\left(\begin{array}{cc}
I_{4} & 0 \\
0 & x \sigma x^{-1}
\end{array}\right)\left(\begin{array}{cc}
0 & y \\
y^{-1} & 0
\end{array}\right)
$$

for some $x$ and $y$ in $\operatorname{Aut}(\mathcal{T})$ from Proposition 2.10. In the first case we can choose an element $z$ in $\operatorname{Aut}(\mathcal{T})$ such that $z=y^{-1} x$. Then

$$
\begin{aligned}
r l a & =\left(\begin{array}{cc}
x \sigma x^{-1} & 0 \\
0 & I_{4}
\end{array}\right)\left(\begin{array}{cc}
0 & y \\
y^{-1} & 0
\end{array}\right) \\
& =\left[\left(\begin{array}{ll}
x & 0 \\
0 & z
\end{array}\right)\left(\begin{array}{cc}
\sigma & 0 \\
0 & I_{4}
\end{array}\right)\left(\begin{array}{cc}
x^{-1} & 0 \\
0 & z^{-1}
\end{array}\right)\right]\left(\begin{array}{cc}
0 & y \\
y^{-1} & 0
\end{array}\right) \\
& =\left[\left(\begin{array}{ll}
x & 0 \\
0 & z
\end{array}\right)\left(\begin{array}{cc}
\sigma & 0 \\
0 & I_{4}
\end{array}\right)\left(\begin{array}{cc}
x^{-1} & 0 \\
0 & z^{-1}
\end{array}\right)\right]\left[\left(\begin{array}{cc}
x & 0 \\
0 & z
\end{array}\right)\left(\begin{array}{cc}
0 & I_{4} \\
I_{4} & 0
\end{array}\right)\left(\begin{array}{cc}
x^{-1} & 0 \\
0 & z^{-1}
\end{array}\right)\right] \\
& =\left(\begin{array}{ll}
x & 0 \\
0 & z
\end{array}\right)\left(\begin{array}{cc}
\sigma & 0 \\
0 & I_{4}
\end{array}\right)\left(\begin{array}{cc}
0 & I_{4} \\
I_{4} & 0
\end{array}\right)\left(\begin{array}{cc}
x^{-1} & 0 \\
0 & z^{-1}
\end{array}\right) .
\end{aligned}
$$

Since $\left(\begin{array}{cc}\sigma & 0 \\ 0 & I_{4}\end{array}\right)\left(\begin{array}{cc}0 & I_{4} \\ I_{4} & 0\end{array}\right)=\phi_{1}(\sigma) \phi_{2}(I) \gamma, a$ is contained in the conjugacy class of $\phi_{1}(\sigma) \phi_{2}(I) \gamma$. For the second case, we take $z$ in $\operatorname{Aut}(\mathcal{T})$ such that $z=y x$. Then

$$
\begin{aligned}
r l a & =\left(\begin{array}{cc}
I_{4} & 0 \\
0 & x \sigma x^{-1}
\end{array}\right)\left(\begin{array}{cc}
0 & y \\
y^{-1} & 0
\end{array}\right) \\
& =\left[\left(\begin{array}{ll}
z & 0 \\
0 & x
\end{array}\right)\left(\begin{array}{cc}
I_{4} & 0 \\
0 & \sigma
\end{array}\right)\left(\begin{array}{cc}
z^{-1} & 0 \\
0 & x^{-1}
\end{array}\right)\right]\left(\begin{array}{cc}
0 & y \\
y^{-1} & 0
\end{array}\right) \\
& =\left[\left(\begin{array}{ll}
z & 0 \\
0 & x
\end{array}\right)\left(\begin{array}{cc}
I_{4} & 0 \\
0 & \sigma
\end{array}\right)\left(\begin{array}{cc}
z^{-1} & 0 \\
0 & x^{-1}
\end{array}\right)\right]\left[\left(\begin{array}{cc}
z & 0 \\
0 & x
\end{array}\right)\left(\begin{array}{cc}
0 & I_{4} \\
I_{4} & 0
\end{array}\right)\left(\begin{array}{cc}
z^{-1} & 0 \\
0 & x^{-1}
\end{array}\right)\right] \\
& =\left(\begin{array}{ll}
z & 0 \\
0 & x
\end{array}\right)\left(\begin{array}{cc}
I_{4} & 0 \\
0 & \sigma
\end{array}\right)\left(\begin{array}{cc}
0 & I_{4} \\
I_{4} & 0
\end{array}\right)\left(\begin{array}{cc}
z^{-1} & 0 \\
0 & x^{-1}
\end{array}\right) .
\end{aligned}
$$


Since $\left(\begin{array}{cc}I_{4} & 0 \\ 0 & \sigma\end{array}\right)\left(\begin{array}{cc}0 & I_{4} \\ I_{4} & 0\end{array}\right)=\phi_{2}(\sigma) \phi_{1}(I) \gamma$, and $\phi_{2}(\sigma) \phi_{1}(I)=\phi_{1}(I) \phi_{2}(\sigma)$, from Proposition 2.10, $a$ is contained in the conjugacy class of $\phi_{1}(\sigma) \phi_{2}(I) \gamma$. Therefore the conjugacy class of $\phi_{1}(\sigma) \phi_{2}\left(I_{4}\right) \gamma$ and $C_{\sigma} C_{\gamma}$ are the same.

If we apply the same argument in Theorem 2.16, the following corollarly is true.

Corollary 2.17. The conjugacy class of $\phi_{1}(\sigma) \phi_{2}\left(-I_{4}\right) \gamma$ is equal to $C_{\sigma} C_{\gamma}$, where $C_{\sigma}$ is the conjugacy class of $\phi_{1}(\sigma) \phi_{2}\left(-I_{4}\right)$ and $C_{\gamma}$ is the conjugacy class of $\gamma$.

The following proposition gives the number of elements of conjugacy class of $\phi_{1}(\sigma) \phi_{2}\left(I_{4}\right) \gamma$.

Proposition 2.18. The number of elements of $C_{\sigma} C_{\gamma}$ is equal to $\frac{1}{2}\left|C_{\sigma} \| C_{\gamma}\right|$, where $C_{\sigma}$ is the conjugacy class of $\phi_{1}(\sigma) \phi_{2}\left(I_{4}\right)$ and $C_{\gamma}$ is the conjugacy class of $\gamma$.

Proof. For any $\left(\begin{array}{cc}I_{4} & 0 \\ 0 & \sigma^{\prime}\end{array}\right) \in C_{\sigma}$ and $x \in \operatorname{Aut}(\mathcal{T})$

$$
\left(\begin{array}{cc}
I_{4} & 0 \\
0 & \sigma^{\prime}
\end{array}\right)\left(\begin{array}{cc}
0 & x \\
x^{-1} & 0
\end{array}\right)=\left(\begin{array}{cc}
0 & x \\
\sigma^{\prime} x^{-1} & 0
\end{array}\right) .
$$

We can choose $\left(\begin{array}{cc}\sigma^{\prime \prime} & 0 \\ 0 & I_{4}\end{array}\right) \in C_{\sigma}$ and $y \in \operatorname{Aut}(\mathcal{T})$ such that

$$
\sigma^{\prime \prime}=x \sigma^{\prime} x^{-1} \quad \text { and } \quad y=x \sigma^{\prime-1}
$$

since $x \sigma^{\prime} x^{-1}$ is a conjugate of $\sigma$. Then

$$
\begin{aligned}
r l\left(\begin{array}{cc}
\sigma^{\prime \prime} & 0 \\
0 & I_{4}
\end{array}\right)\left(\begin{array}{cc}
0 & y \\
y^{-1} & 0
\end{array}\right) & =\left(\begin{array}{cc}
0 & \sigma^{\prime \prime} y \\
y^{-1} & 0
\end{array}\right) \\
& =\left(\begin{array}{cc}
0 & \left(x \sigma^{\prime} x^{-1}\right)\left(x \sigma^{\prime-1}\right) \\
\left(x \sigma^{\prime-1}\right)^{-1} & 0
\end{array}\right) \\
& =\left(\begin{array}{cc}
0 & x \\
\sigma^{\prime} x^{-1} & 0
\end{array}\right) .
\end{aligned}
$$

Hence we obtain the products of the form of $\left(\begin{array}{cc}I_{4} & 0 \\ 0 & \frac{\sigma}{\sigma}\end{array}\right)\left(\begin{array}{cc}0 & x \\ x^{-1} & 0\end{array}\right)$ by the products of the form of $\left(\begin{array}{cc}\bar{\sigma} & 0 \\ 0 & I_{4}\end{array}\right)\left(\begin{array}{cc}0 & x \\ x^{-1} & 0\end{array}\right)$.

Suppose that

$$
\left(\begin{array}{cc}
\sigma_{1} & 0 \\
0 & I_{4}
\end{array}\right)\left(\begin{array}{cc}
0 & x \\
x^{-1} & 0
\end{array}\right)=\left(\begin{array}{cc}
\sigma_{2} & 0 \\
0 & I_{4}
\end{array}\right)\left(\begin{array}{cc}
0 & y \\
y^{-1} & 0
\end{array}\right) .
$$

Then we should have $x=y$ and $\sigma_{1}=\sigma_{2}$. Therefore we get the desired result $\left|C_{\sigma} C_{\gamma}\right|=\frac{1}{2}\left|C_{\sigma} \| C_{\gamma}\right|$.

We can compute the number of elements of conjugacy class of $\phi_{1}(\sigma) \phi_{2}\left(-I_{4}\right) \gamma$ using the same argument in Proposition 2.18. 
Corollary 2.19. The number of elements of $C_{\sigma} C_{\gamma}$ is equal to $\frac{1}{2}\left|C_{\sigma} \| C_{\gamma}\right|$, where $C_{\sigma}$ is the conjugacy class of $\phi_{1}(\sigma) \phi_{2}\left(-I_{4}\right)$ and $C_{\gamma}$ is the conjugacy class of $\gamma$.

Proof. For any $\left(\begin{array}{cc}-I_{4} & 0 \\ 0 & \sigma^{\prime}\end{array}\right) \in C_{\sigma}$ and $x \in \operatorname{Aut}(\mathcal{T})$, we have

$$
\left(\begin{array}{cc}
-I_{4} & 0 \\
0 & \sigma^{\prime}
\end{array}\right)\left(\begin{array}{cc}
0 & x \\
x^{-1} & 0
\end{array}\right)=\left(\begin{array}{cc}
0 & -x \\
\sigma^{\prime} x^{-1} & 0
\end{array}\right)
$$

We can choose $\left(\begin{array}{cc}\sigma^{\prime \prime} & 0 \\ 0 & -I_{4}\end{array}\right) \in C_{\sigma}$ and $y \in \operatorname{Aut}(\mathcal{T})$ such that

$$
\sigma^{\prime \prime}=-x \sigma^{\prime} x^{-1} \text { and } y=x \sigma^{\prime-1} \text {, }
$$

since $x \sigma^{\prime} x^{-1}$ is a conjugate of $\sigma$. Then we have

$$
\begin{aligned}
r l\left(\begin{array}{cc}
\sigma^{\prime \prime} & 0 \\
0 & I_{4}
\end{array}\right)\left(\begin{array}{cc}
0 & y \\
y^{-1} & 0
\end{array}\right) & =\left(\begin{array}{cc}
0 & \sigma^{\prime \prime} y \\
y^{-1} & 0
\end{array}\right) \\
& =\left(\begin{array}{cc}
0 & \left(-x \sigma^{\prime} x^{-1}\right)\left(x \sigma^{\prime-1}\right) \\
\left(x \sigma^{\prime-1}\right)^{-1} & 0
\end{array}\right) \\
& =\left(\begin{array}{cc}
0 & -x \\
\sigma^{\prime} x^{-1} & 0
\end{array}\right) .
\end{aligned}
$$

This completes the statement.

Let us show the result in Table 1 , where $C_{i}$ is in [Table 2, [4]]. From this Table 1, we obtain the class equation for $\mathbf{G}$ :

$$
\sum_{i=1}^{44} C_{i}=4608 .
$$

Lemma 2.20 ([4, Theorems 3.3 and 3.4]). The automorphism group of $\mathcal{T}$ has four Sylow 3-subgroups of order 3 and three Sylow 2-subgroups of order 16 . Thus no Sylow subgroups are normal.

We consider the Sylow subgroups of $\mathcal{C}$. The results about Sylow subgroups are the following.

Theorem 2.21. The automorphism group of $\mathcal{C}$ has 9 Sylow 2-subgroups of order 512 and 16 Sylow 3-subgroups of order 9 .

Proof. Let $S$ be a Sylow 2-subgroup of G. It is obvious that the order of $S$ is 512. Since 512 does not divide the order of $\mathbf{G}_{1} \mathbf{G}_{2}, S$ can not be the form of $X Y$ for some $X \leq \mathbf{G}_{1}, Y \leq \mathbf{G}_{2}$. Now, we consider the normalizer of $\phi_{1}\left(P_{i}\right) \phi_{2}\left(P_{j}\right)$ for $i, j=1,2,3$, where $P_{1}, P_{2}$, and $P_{3}$ are Sylow 2-subgroups of $\operatorname{Aut}(\mathcal{T})$ in [4]. Since $\left|\phi_{1}\left(P_{i}\right) \phi_{2}\left(P_{j}\right)\right|$ is 256 , its normalizer must contain some Sylow 2-subgroup from the first Sylow theorem in [3]. Since $P_{1}, P_{2}$ and $P_{3}$ are distinct Sylow 2subgroups of $\operatorname{Aut}(\mathcal{T})$ and $|\operatorname{Aut}(\mathcal{T})|=48$, one should have $N_{\text {Aut }(\mathcal{T})}\left(P_{i}\right)=P_{i}$ for $i=1,2,3$. Therefore, $\phi_{1}\left(P_{i}\right) \phi_{2}\left(P_{j}\right)$ is not a normal subgroup of $\mathbf{G}_{1} \mathbf{G}_{2}$, and hence its normalizer does't have an element of order 3 . Consequently, the normalizer of $\phi_{1}\left(P_{i}\right) \phi_{2}\left(P_{j}\right)$ has order 512 from the first Sylow theorem in [3]. 
Since $N_{\operatorname{Aut}(\mathcal{T})}\left(P_{i}\right)$ is not equal to $N_{\operatorname{Aut}(\mathcal{T})}\left(P_{j}\right)$ where $i$ and $j$ are distinct, we obtain distinct 9 subgroups of order 512. They are Sylow 2-subgroups of $\mathbf{G}$. Let $N_{2}$ be the number of Sylow 2-subgroups of G. By Sylow theorems, we should have $N_{2} \equiv 1(\bmod 2)$ and $N_{2} \mid 9$. However, the possible numbers for $N_{2}$ are 1,3 , or 9 and we already show there exist only 9 Sylow 2 -subgroups. Hence G has only 9 Sylow 2-subgroups.

Each element of $\mathbf{G}$ can be written uniquely as a product $g_{1} g_{2} g_{3}$ for some $g_{1} \in$ $\mathbf{G}_{1}, g_{2} \in \mathbf{G}_{2}$, and $g_{3} \in \mathbf{G}_{3}$. The fact that the order of $g_{1} g_{2} g_{3}$ divides 9 implies $g_{3}=I_{8}$. Hence $\phi_{1}\left(Q_{i}\right) \phi_{2}\left(Q_{j}\right)$ are Sylow 3-subgroups of $\mathbf{G}$ for $i=1,2,3,4$ and $j=1,2,3,4$, where $Q_{1}, Q_{2}, Q_{3}$, and $Q_{4}$ in [4]. Since there is no element of $\mathbf{G}_{1} \mathbf{G}_{2}$ whose its order is 9 , only these 16 subgroups are Sylow 3 -subgroups.

TABLE 1. Conjugacy classes of $\mathbf{G}$

\begin{tabular}{|c|c|c|c|c|}
\hline Class & Representative & Elements & Length & Order \\
\hline$C_{1}$ & $\phi_{1}(1) \phi_{2}(1)$ & $\phi_{1}(C 1) \phi_{2}(C 1)$ & 1 & 1 \\
\hline$C_{2}$ & $\phi_{1}(48) \phi_{2}(48)$ & $\phi_{1}(C 8) \phi_{2}(C 8)$ & 1 & 2 \\
\hline$C_{3}$ & $\phi_{1}(48) \phi_{2}(1)$ & $\phi_{1}(C 8) \phi_{2}(C 1) \cup \phi_{1}(C 1) \phi_{2}(C 8)$ & 2 & 2 \\
\hline$C_{4}$ & $\phi_{1}(48) \phi_{2}(6)$ & $\phi_{1}(C 8) \phi_{2}(C 3) \cup \phi_{1}(C 3) \phi_{2}(C 8)$ & 24 & 2 \\
\hline$C_{5}$ & $\phi_{1}(1) \phi_{2}(43)$ & $\phi_{1}(C 1) \phi_{2}(C 3) \cup \phi_{1}(C 3) \phi_{2}(C 1)$ & 24 & 2 \\
\hline$C_{6}$ & $\gamma$ & (2) in Proposition 2.12 & 48 & 2 \\
\hline$C_{7}$ & $\phi_{1}(3) \phi_{2}(3)$ & $\phi_{1}(C 3) \phi_{2}(C 3)$ & 144 & 2 \\
\hline$C_{8}$ & $\phi_{1}(1) \phi_{2}(4)$ & $\phi_{1}(C 1) \phi_{2}(C 2) \cup \phi_{1}(C 2) \phi_{2}(C 1)$ & 16 & 3 \\
\hline$C_{9}$ & $\phi_{1}(2) \phi_{2}(4)$ & $\phi_{1}(C 2) \phi_{2}(C 2)$ & 64 & 3 \\
\hline$C_{10}$ & $\phi_{1}(1) \phi_{2}(27)$ & $\phi_{1}(C 1) \phi_{2}(C 7) \cup \phi_{1}(C 7) \phi_{2}(C 1)$ & 12 & 4 \\
\hline$C_{11}$ & $\phi_{1}(48) \phi_{2}(22)$ & $\phi_{1}(C 8) \phi_{2}(C 7) \cup \phi_{1}(C 7) \phi_{2}(C 8)$ & 12 & 4 \\
\hline$C_{12}$ & $\phi_{1}(37) \phi_{2}(27)$ & $\phi_{1}(C 7) \phi_{2}(C 7)$ & 36 & 4 \\
\hline$C_{13}$ & $\phi_{1}(48) \phi_{2}(1) \gamma$ & $C_{3} C_{6}$ & 48 & 4 \\
\hline$C_{14}$ & $\phi_{1}(37) \phi_{2}(6)$ & $\phi_{1}(C 7) \phi_{2}(C 3) \cup \phi_{1}(C 3) \phi_{2}(C 7)$ & 144 & 4 \\
\hline$C_{15}$ & $\phi_{1}(1) \phi_{2}(43) \gamma$ & $C_{5} C_{6}$ & 576 & 4 \\
\hline$C_{16}$ & $\phi_{1}(48) \phi_{2}(4)$ & $\phi_{1}(C 8) \phi_{2}(C 2) \cup \phi_{1}(C 2) \phi_{2}(C 8)$ & 16 & 6 \\
\hline$C_{17}$ & $\phi_{1}(48) \phi_{2}(45)$ & $\phi_{1}(C 8) \phi_{2}(C 6) \cup \phi_{1}(C 6) \phi_{2}(C 8)$ & 16 & 6 \\
\hline$C_{18}$ & $\phi_{1}(1) \phi_{2}(45)$ & $\phi_{1}(C 1) \phi_{2}(C 6) \cup \phi_{1}(C 6) \phi_{2}(C 1)$ & 16 & 6 \\
\hline$C_{19}$ & $\phi_{1}(47) \phi_{2}(45)$ & $\phi_{1}(C 6) \phi_{2}(C 6)$ & 64 & 6 \\
\hline$C_{20}$ & $\phi_{1}(47) \phi_{2}(4)$ & $\phi_{1}(C 6) \phi_{2}(C 2) \cup \phi_{1}(C 2) \phi_{2}(C 6)$ & 128 & 6 \\
\hline$C_{21}$ & $\phi_{1}(2) \phi_{2}(44)$ & $\phi_{1}(C 2) \phi_{2}(C 3) \cup \phi_{1}(C 3) \phi_{2}(C 2)$ & 192 & 6 \\
\hline$C_{22}$ & $\phi_{1}(47) \phi_{2}(5)$ & $\phi_{1}(C 6) \phi_{2}(C 3) \cup \phi_{1}(C 3) \phi_{2}(C 6)$ & 192 & 6 \\
\hline$C_{23}$ & $\phi_{1}(1) \phi_{2}(4) \gamma$ & $C_{8} C_{6}$ & 384 & 6 \\
\hline$C_{24}$ & $\phi_{1}(1) \phi_{2}(33)$ & $\phi_{1}(C 1) \phi_{2}(C 4) \cup \phi_{1}(C 4) \phi_{2}(C 1)$ & 12 & 8 \\
\hline$C_{25}$ & $\phi_{1}(48) \phi_{2}(16)$ & $\phi_{1}(C 8) \phi_{2}(C 5) \cup \phi_{1}(C 5) \phi_{2}(C 8)$ & 12 & 8 \\
\hline$C_{26}$ & $\phi_{1}(1) \phi_{2}(16)$ & $\phi_{1}(C 1) \phi_{2}(C 5) \cup \phi_{1}(C 5) \phi_{2}(C 1)$ & 12 & 8 \\
\hline$C_{27}$ & $\phi_{1}(48) \phi_{2}(33)$ & $\phi_{1}(C 8) \phi_{2}(C 4) \cup \phi_{1}(C 4) \phi_{2}(C 8)$ & 12 & 8 \\
\hline$C_{28}$ & $\phi_{1}(25) \phi_{2}(41)$ & $\phi_{1}(C 5) \phi_{2}(C 5)$ & 36 & 8 \\
\hline
\end{tabular}


TABLE 1. continued

\begin{tabular}{|c|c|c|c|c|}
\hline$C_{29}$ & $\phi_{1}(24) \phi_{2}(8)$ & $\phi_{1}(C 4) \phi_{2}(C 4)$ & 36 & 8 \\
\hline$C_{30}$ & $\phi_{1}(12) \phi_{2}(33)$ & $\phi_{1}(C 7) \phi_{2}(C 4) \cup \phi_{1}(C 4) \phi_{2}(C 7)$ & 72 & 8 \\
\hline$C_{31}$ & $\phi_{1}(24) \phi_{2}(41)$ & $\phi_{1}(C 4) \phi_{2}(C 5) \cup \phi_{1}(C 5) \phi_{2}(C 4)$ & 72 & 8 \\
\hline$C_{32}$ & $\phi_{1}(37) \phi_{2}(16)$ & $\phi_{1}(C 7) \phi_{2}(C 5) \cup \phi_{1}(C 5) \phi_{2}(C 7)$ & 72 & 8 \\
\hline$C_{33}$ & $\phi_{1}(46) \phi_{2}(25)$ & $\phi_{1}(C 3) \phi_{2}(C 5) \cup \phi_{1}(C 5) \phi_{2}(C 3)$ & 144 & 8 \\
\hline$C_{34}$ & $\phi_{1}(3) \phi_{2}(24)$ & $\phi_{1}(C 3) \phi_{2}(C 4) \cup \phi_{1}(C 4) \phi_{2}(C 3)$ & 144 & 8 \\
\hline$C_{35}$ & $\phi_{1}(1) \phi_{2}(27) \gamma$ & $C_{10} C_{6}$ & 288 & 8 \\
\hline$C_{36}$ & $\phi_{1}(37) \phi_{2}(14)$ & $\phi_{1}(C 7) \phi_{2}(C 2) \cup \phi_{1}(C 2) \phi_{2}(C 7)$ & 96 & 12 \\
\hline$C_{37}$ & $\phi_{1}(12) \phi_{2}(35)$ & $\phi_{1}(C 7) \phi_{2}(C 6) \cup \phi_{1}(C 6) \phi_{2}(C 7)$ & 96 & 12 \\
\hline$C_{38}$ & $\phi_{1}(48) \phi_{2}(4) \gamma$ & $C_{16} C_{6}$ & 384 & 12 \\
\hline$C_{39}$ & $\phi_{1}(1) \phi_{2}(16) \gamma$ & $C_{26} C_{6}$ & 288 & 16 \\
\hline$C_{40}$ & $\phi_{1}(48) \phi_{2}(16) \gamma$ & $C_{25} C_{6}$ & 288 & 16 \\
\hline$C_{41}$ & $\phi_{1}(47) \phi_{2}(13)$ & $\phi_{1}(C 6) \phi_{2}(C 4) \cup \phi_{1}(C 4) \phi_{2}(C 6)$ & 96 & 24 \\
\hline$C_{42}$ & $\phi_{1}(2) \phi_{2}(36)$ & $\phi_{1}(C 2) \phi_{2}(C 5) \cup \phi_{1}(C 5) \phi_{2}(C 2)$ & 96 & 24 \\
\hline$C_{43}$ & $\phi_{1}(47) \phi_{2}(36)$ & $\phi_{1}(C 6) \phi_{2}(C 5) \cup \phi_{1}(C 5) \phi_{2}(C 6)$ & 96 & 24 \\
\hline$C_{44}$ & $\phi_{1}(2) \phi_{2}(13)$ & $\phi_{1}(C 2) \phi_{2}(C 4) \cup \phi_{1}(C 4) \phi_{2}(C 2)$ & 96 & 24 \\
\hline
\end{tabular}

We define $i$ and $j$ of $\phi_{1}(i) \phi_{2}(j)$ as $j$ in [Table 1, [4]].

\section{References}

[1] Thomas W. Hugerford, Algebra, Springer-Verlag, New york, 1974.

[2] C. L. Mallows, V. Pless, and N. J. A. Sloane, Self-dual codes over GF(3), SIAM J. Appl. Math. 21 (1976), no. 4, 649-666.

[3] C. L. Mallows and N. J. A. Sloane, An upper bound for self-dual codes, Inform. and Control 22 (1973), 188-200.

[4] Y. H. Park, Automorphism group of the ternary tetracode, Korean J. Math. 17 (2009), no. 4, 487-493.

[5] Eric M. Rains and N. J. A. Sloane, Self-dual codes, in: V. S. Pless and W. C. Huffman (Eds.), Handbook of coding theory, Vol. I, II, 177294, North-Holland, Amsterdam, 1998.

HYUN JiN KIM

Institute of Mathematical SCIENCES

EWHa Womans University

SEOUL 120-750, KoreA

E-mail address: guswls41@ewha.ac.kr

June BoK LEe

Department of Mathematics

YONSEI UNIVERSITY

SeOUl 120-749, Korea

E-mail address: leejb@yonsei.ac.kr 